\title{
The right to independent living for adult people with disabilities and emancipatory opportunities in Italy
}

\author{
Luca Decembrotto*
}

\begin{abstract}
The present contribution proposes some reflections stemming from the right to independent living for adult people with disabilities and from the level of emancipation, lingering on some specific housing projects available in Italy. The examined data comes from a nationwide research carried out by the University of Bologna, which focused on which residential services are available today for adult people with disabilities, taking also in account how stimulation extends towards the dwellers in choosing autonomous housing.
\end{abstract}

Keywords: disability, housing, independent living

\section{The Right to Housing}

In contemporary culture, the housing dimension is a cornerstone on the way to self-determination and the emancipatory process of a person, a true element of transition toward adulthood to express freedom of choice and the faculty to imagine the future and plan it (Lepri, 2011).

The place called "home" can be defined from several concepts linked with space, which highlights its multidimensionality: the physical, social and legal spaces (Edgar and Meert, 2005; Edgar, Meert and Doherty, 2004). For "physical space" we intend the need to have a dignified home, capable to satisfy personal and familial necessities in terms of living space; the "social space" refers to the possibility to maintain the personal privacy and, at the same time, to enjoy interpersonal relationships; however, the "legal space" outlines the exclusive use, safety and the validity of the legal housing contract. Thanks to the Universal Declaration of Human Rights of 1948, we can speak of the right to housing (or the right to a house), recognised as a fundamental right in Article 245, comma 2:

«Everyone has the right to a standard of living adequate for the health and well-being of himself and of his family, including food, clothing, housing and

* Assegnista di ricerca presso il Dipartimento di Scienze dell'educazione "Giovanni Maria Bertin” dell’Università degli Studi di Bologna. E-mail: luca.decembrotto@unibo.it. 
medical care and necessary social services, and the right to security in the event of unemployment, sickness, disability, widowhood, old age or other lack of livelihood in circumstances beyond his control».

Nevertheless, this right, albeit recognised as a universal human right, is also a "fragile" right, largely unattended among the most marginalised and poor segments of the population (it is enough to think to the homeless) who need public policies to grant it, especially in times of economic or housing crisis. According to the Fourth Overview Of Housing Exclusion In Europe 2019, carried out by Feantsa and Foundation Abbé Pierre (2019), an approximate $10,4 \%$ of the European Union's population (23.017.924 families) spends more than $40 \%$ of the family income of housing costs and approximately $4 \%$ (8.853.048 families) lives in inadequate lodgings, with 700,000 people who live on the streets every night (a 70\% increase compared to 2009).

Often, the missing recognition of this right involves people with disabilities: it is enough to think that in nearly all the member countries of the European Union there are no basic set standards of accessibility to the structures or the emergency lodgings for the homeless or the people with reduced mobility. However, their involvement is not limited only to the issues that concern poverty and housing deprivation. In many cultural contexts, leaving the nuclear family is not perceived as a necessary transition, to be stimulated, encouraged and realised with specific supports leading to a gradual autonomous living for the subject with disabilities. In real terms, this process is postponed or, sometimes, denied, affecting the realisation of the personal project. Therefore, if conquering this autonomy could be considered as a past struggle, for some "categories" of people this is still an uphill struggle, despite the existence of the right to live within the communities with the same degree of freedom of choice of all the community members (the principle of equality). This principle is confirmed also in the Convention on the Right of Persons with Disabilities, Article 19, comma 2, dedicated to "Living independently and being included in the community":

"Persons with disabilities have the opportunity to choose their place of residence and where and with whom they live on an equal basis with others and are not obliged to live in a particular living arrangement».

Implementing this right brings some initial considerations. A housing right that protects the people with disabilities requires an ample reflection on accessibility, which includes, transversally, all the environmental factors, all those aspects of the physical and social worlds and those attitudes (WHO, 2001) that may obstacle or compromise the fruition of the spaces. In this case, the socio-pedagogical action aims to transform these aspects into facilitating factors, so that all spaces including the living one, can be reached and used 
independently. The availability of a place where to live, its reachability, access, safety in all its aspects, including its furniture, represent an important aspect on which everyone, even those with disabilities, builds his/her quality of life and well-being (d'Alonzo, 2009). The structuring of a Life Project cannot ignore this dimension or consider it only as extrema ratio, a resource to be activated when other solutions (usually provided by the family) are not viable. Outside the boundaries of the right, in the present cultural and social context, independent housing is also the expression of the adulthood of the person, a condition strictly connected to the person's maturity and ability in making choices; an essential passage in recognising the adult life of people with disabilities.

\section{Disability and independent living}

The missing access to the right of housing increases the difficulties that people with disability experience already with the acknowledgement of their "adulthood" (Caldin and Friso, 2012; Lepri, 2011; Goussot, 2009), an acknowledgment (precisely, a process of acknowledgement) that should not only assume the biological development that comes with adult age, but extended to the psychological and socio-relational changes, taking into account also the needs, aspirations and the possibility to try different roles of the person (Friso, 2017), to encourage the development of the identity of the person. When it comes to housing, it is a question of recognising the possibility of living where and with whomever you want, thus having the opportunity to take care of your relationships, moments of personal fulfilment and acquire skills for the widest possible autonomy, providing an answer to the personal and social needs of the person within the spatial and temporal dimensions typical of living, according to a wider interpretation of living not limited to its mere architectural reference.

Recognising the "adulthood" of the person with disabilities means overcoming all those social representations that denied this possibility. For a long time, discriminatory expressions such like handicapped or abnormal carried with them the idea that the person with disabilities should be segregated and kept away as much as possible in places of care oriented to the publicly recognised needs (only these needs); and the person should be subordinated to somebody else's will. A real process of marginalisation took place, it has not yet been completely overcome. The family, the most important primary social educational agent, had and continues to have a central role in making possible to start these alternative paths. It is the first context where a person experiences the social reality, experiencing success and failure, which contribute meaningfully to his/her education and growth. The family has an essential 
function in the process of recognition: in fact, it can play the role of the promoter of the autonomy, feeding the construction of a positive self-image and sustaining the gradual development of self-determination. However, the family can also play a role that opposes the emancipatory process, avoiding to separate from the son or daughter with disabilities after the multiple difficulties experienced in many occasions in dealing with the outside world and, at the same time, feeding the construction a strongly hetero-directed self-image, fragile and dependent on the others. In the same way, the context can hinder or ease the gradual maturation of the subject with disabilities according to the degree of recognition given, as self-representation undergoes also through all the representations that groups and communities elaborate on their members and the social roles assigned to them.

Not guaranteeing persons with disabilities the right to choose their own home applying the principle of equality and by placing this right before traditional responses such as specialized institutes or centres, can hide not only discriminatory elements based on an institutionalising approach, where the person with disabilities may feel alienated from the possibility of handling his/her body and person, but it also contains new forms of segregation. The exclusion from relationships and/or contacts with other members of the community and their later isolation are always possible events, especially when the other (in this case, the subject with disabilities) is not recognised as a responsible and adult person. On the contrary, the pedagogy for inclusion believes that the person with disabilities should be able to choose and act independently when inserted in a context that considers and recognises him/her as an adult and provides the possibility to access the eventual necessary supports. This is possible thanks to the overcoming of the idea of the condition of disability as a limit or deficiency and with an active of a supporting network, allowing the person to choose, act and realise his/her Life Project, starting from the acquisition of the daily skills needed for the autonomy (Caldin, Montuschi and Canevaro, 2016).

The planning of a Life Project, built around the person and the housing dimension, can avoid the trap of services built upon «a number of redundant and fragmented programmes (often reduced to mere techniques) where everybody is authorised to intervene and where, often, there is no direction of meaning or finality» (Lepri, 2011, p. 89). In fact, the limits of residential experiences are not found in the presence of operators inside the apartment and in their actions (for example, in the forms of assisted living). They are found in the orientation of the socio-educational action towards the well-being of the disabled persons, verifiable in the ability to give space to the decision-making expression on the part of the latter, who, finding themselves in situations of lack or absence of these spaces, will struggle to formulate and share their own Life Project, primarily or exclusively having to fit to other people's projects. 


\section{A nationwide research on the housing experiences of the people with disabilities}

The research introduced here, Indagine sull'autonomia abitativa in Italia (enquiry on the housing independence in Italy), was carried out by the group of Dr Valeria Friso of the University of Bologna in 2016. It was made of an anonymous questionnaire, validated in the group phase; it was addressed to operators managing residential structures for people with disabilities (social cooperatives, foundations, caregiving companies or public agencies, associations) scattered in the country. The research collected data from 97 structures prevalently based in Central and Northern Italy (Table 1). Especially Lombardy, Emilia Romagna and Veneto.

Table 1 - Location of the structures

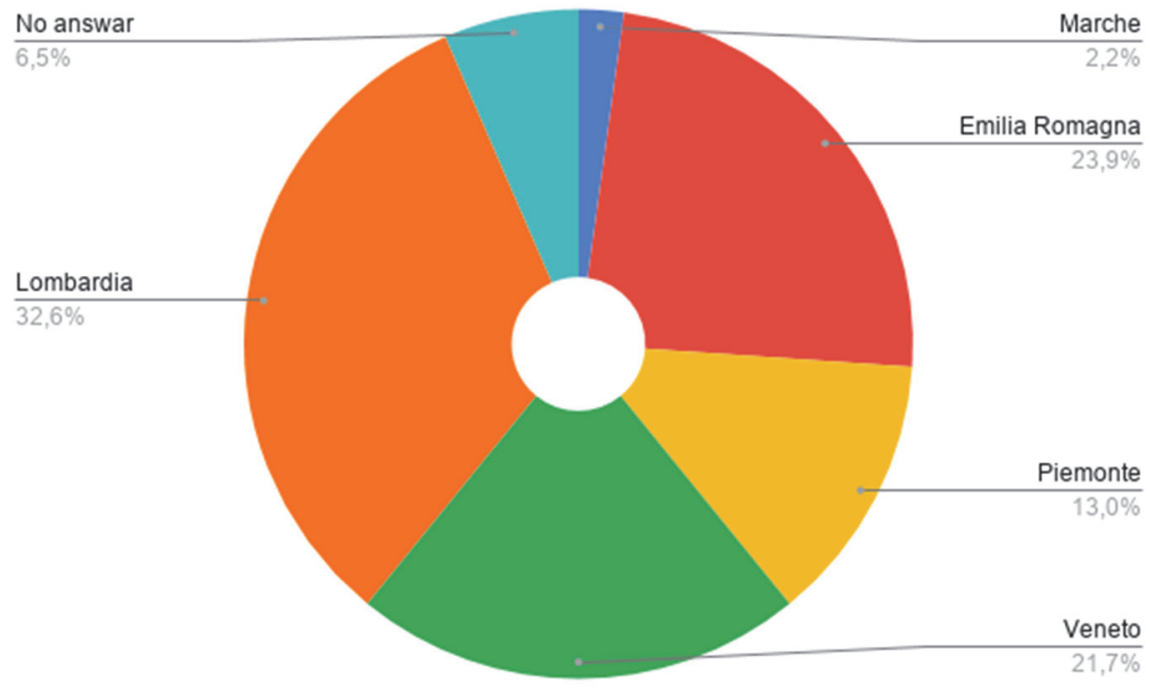

Each analysed institution manages from one to eight structures (Table 2), most of them have one $(40.2 \%)$ or two $(25.8 \%)$ structures, mostly apartments with an average occupancy of 5.1 places each. All together the occupational potential is of approximately 500 places, present occupation rate is of $89 \%$. In the 97 residential structures of this research, in six of them the disabled person lives alone in the apartment, while in five cases the cohabitation concerns ten persons (Table 3). 
Table 2 - Number of apartments per Institution

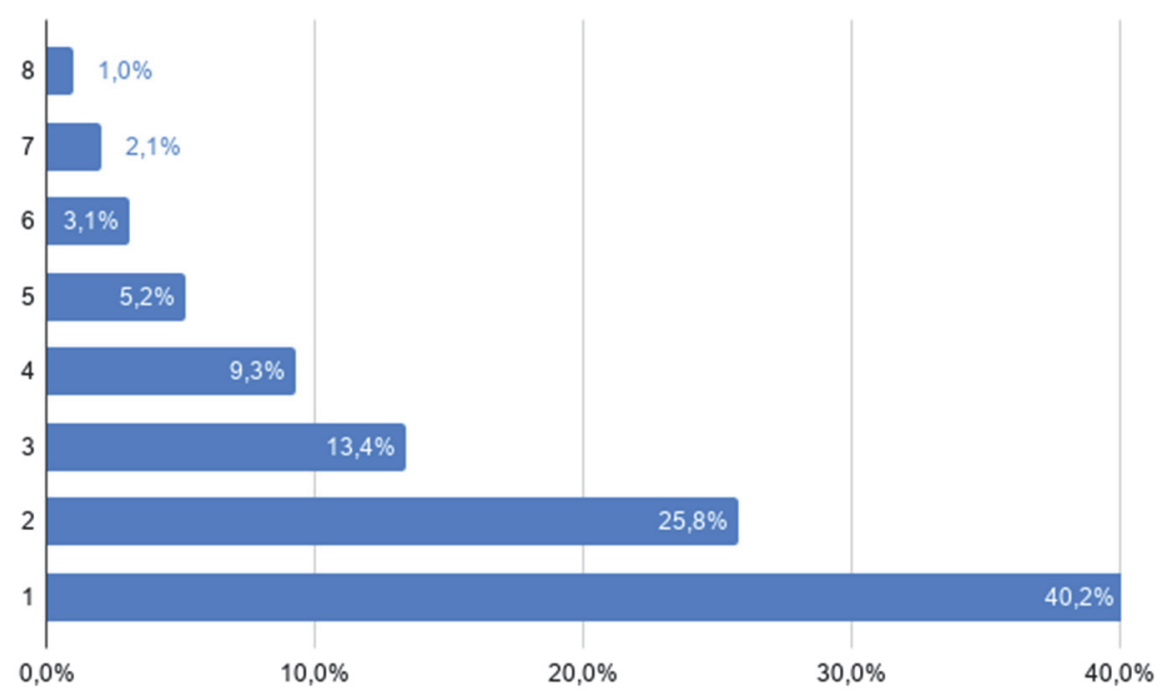

Table 3 - Number of apartments per number of tenants

30

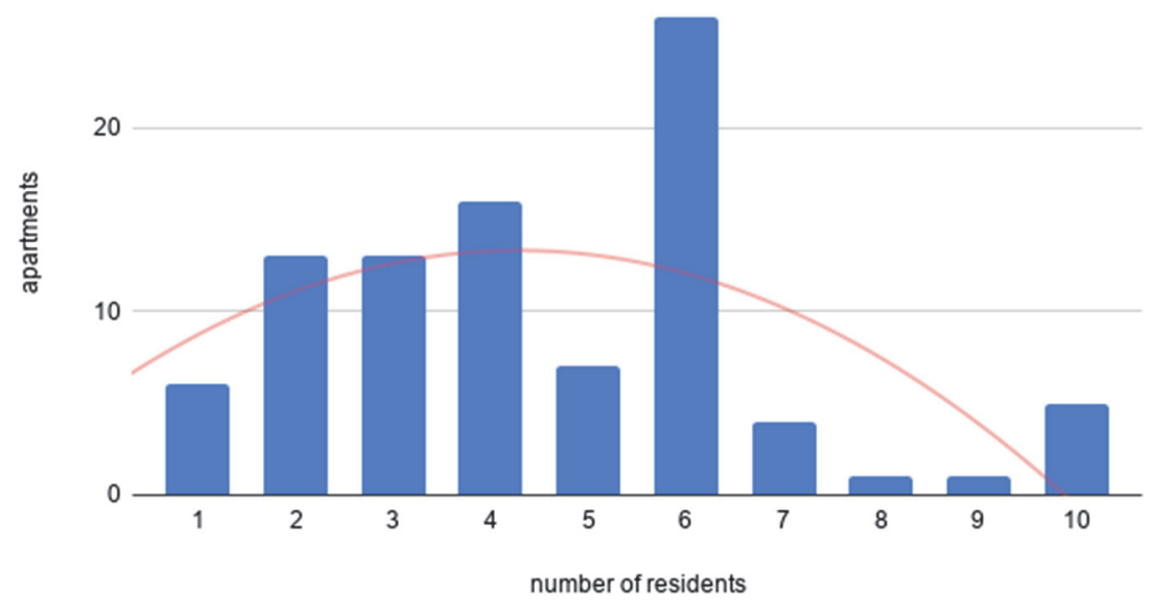

The apartment groups analysed are structured according to permanent residency $(78.3 \%)$, apart from some, fewer, cases where the housing is limited 
to the weekend $(6.5 \%)$, some week per year $(8.7 \%)$ or just one week $(6.5 \%)$ as support to the daily life lived elsewhere.

The professional figures in the residences are mostly educators (33.6\%), and health workers (29.1\%), among them are nurses, health assistants, physiotherapists and more, supported by volunteers (18.2\%). Other significant profiles, but with less impact, are social workers, psychologists, family assistants and social assistance assistants (altogether 16.4\%), while present family members are only $2.7 \%$.

Taking into account all the professional figures whose presence alternates in the apartments, in $41.3 \%$ of the cases we find an all-day-presence and only rarely $(15.2 \%)$ is there a presence with a limited educational or assistive intensity (from 1 to 5 hours daily).

Prospectus 1: average assistive time in the apartments per day

\begin{tabular}{|l|r|}
\hline From 1 to 5 hours & $15.2 \%$ \\
\hline From 6 to 10 hours & $17.4 \%$ \\
\hline From 11 to 15 hours & $13.0 \%$ \\
\hline From 16 to 20 hours & $2.2 \%$ \\
\hline All-day & $41.3 \%$ \\
\hline Other & $6.5 \%$ \\
\hline No answer & $4.4 \%$ \\
\hline
\end{tabular}

The apartments are often owned by the institution (39.1\%), on loan $(23.9 \%)$, or rented $(10.9 \%)$. In the presence of multiple properties managed by the same institution, mixed solutions (8.7\%) are provided for the three types (Table 4). The economic sustainability of the projects is guaranteed through the sharing of expenses by the occupants (26.8\%), the families of the occupants $(24.8 \%)$, the use of the care funds $(4.1 \%)$, the funds from the same cooperative $(7.2 \%)$, the public agency of reference (33.0\%), or other sources (4.1\%). In the case of economical participation from local public agencies these are municipalities, local health agencies, or a mix of these two figures; very rarely, these are the personal care agencies.

The survey examined the stories of 38 people with disabilities involved in these projects, 19 females, 18 males ( 1 unanswered), with an average age of 43.5 years, the youngest was 24 while the oldest was 67 years old. $60.5 \%$ of this group live in an apartment (or structure) throughout the week, only 5.3\% has a "residual" experience in relation to a routine spent elsewhere. $13.2 \%$ have other experiences, such like monthly experiences or "short" weeks. $21.0 \%$ of the sample gave no answer to this question. 
Table 4 - Type of structures used

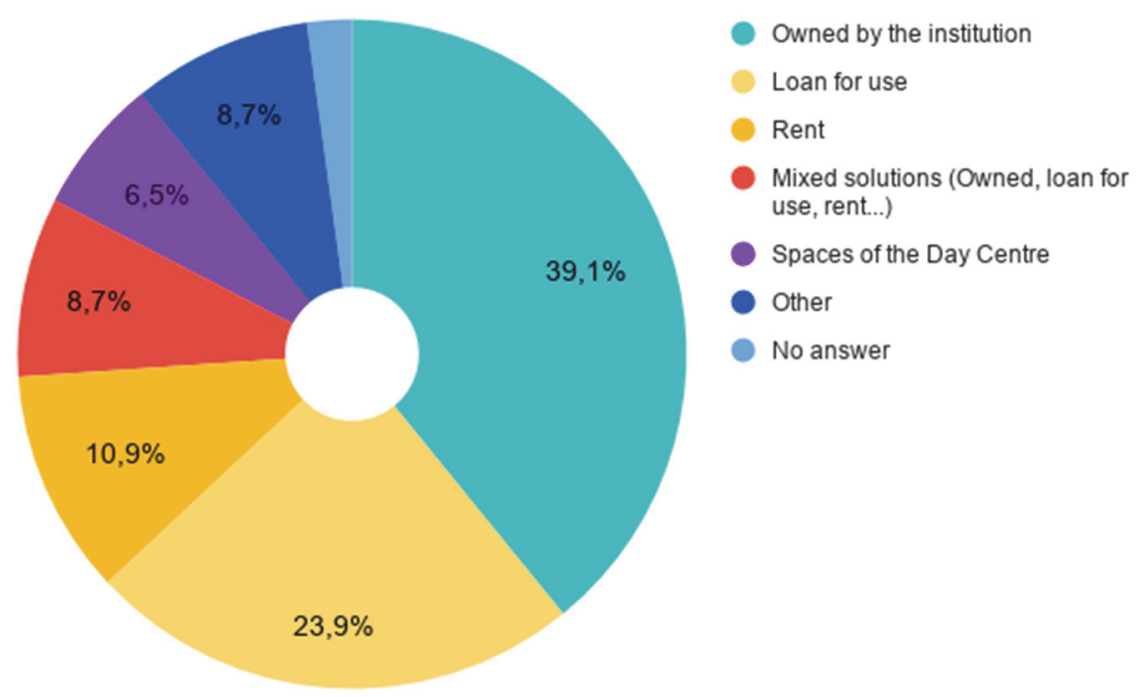

Prospectus 2: Disabilities in the diagnoses

\begin{tabular}{|l|r|}
\hline Down Syndrome & $34.2 \%$ \\
\hline Mild-Moderate Intellectual Disability & $18.4 \%$ \\
\hline Severe Intellectual Disability & $15.8 \%$ \\
\hline Paresis (tetraparesis or paraparesis) & $10.6 \%$ \\
\hline Schizophrenia & $2.6 \%$ \\
\hline $\begin{array}{l}\text { Cervical hydrocephalus, Arnold Chiari Syndrome, Corpus Callosum Hypoplasia, mild } \\
\text { mental retardation }\end{array}$ & $2.6 \%$ \\
\hline $\begin{array}{l}\text { Severe psychic hypoevolutism. Complex partial epilepsy. Grave visual impairment due to } \\
\text { hypermetropia astigmatism with strabismus and amblyopia }\end{array}$ & $2.6 \%$ \\
\hline Intellectual disability with forms of addiction (alcohol) & $2.6 \%$ \\
\hline No answer & $10.6 \%$ \\
\hline
\end{tabular}

In the second half of the questionnaire, the research group investigated the multiple aspects directly connected to the wishes of the people with disabilities involved in the survey and to their perception related to the experience and the acquired competence. Among the items found in this part of the measuring instrument, it was asked to quantify the wish to leave the nuclear family to live independently, on a scale from 1 (no wish) to 4 (very high wish). According to the perception of those who provided the research data, more than half of people 
with disabilities in housing autonomy projects $(57.1 \%)$ are very keen to live their independence (Table 5). Such wish is manifested in different modes:

- through a specific route before entering the apartment, carried out with professionals not immediately involved in the housing project (e.g. local social worker, psychologist, etc.);

- through adherence to the activities of an independent life, from getting satisfaction to carry out some daily activities related to home management, to building and maintaining one's own social network;

- perceiving and representing the living context as home;

- Referring to the previous condition of malaise experienced in the family of origin and expressing the will not to live it anymore or manifesting the will that the moments of return to the family be temporally short;

- Consolidating the social relationships in the apartment and considering the other apartment mates as reference points.

Table 5 - Degree of desire to leave the nuclear family

$$
60,0 \%
$$

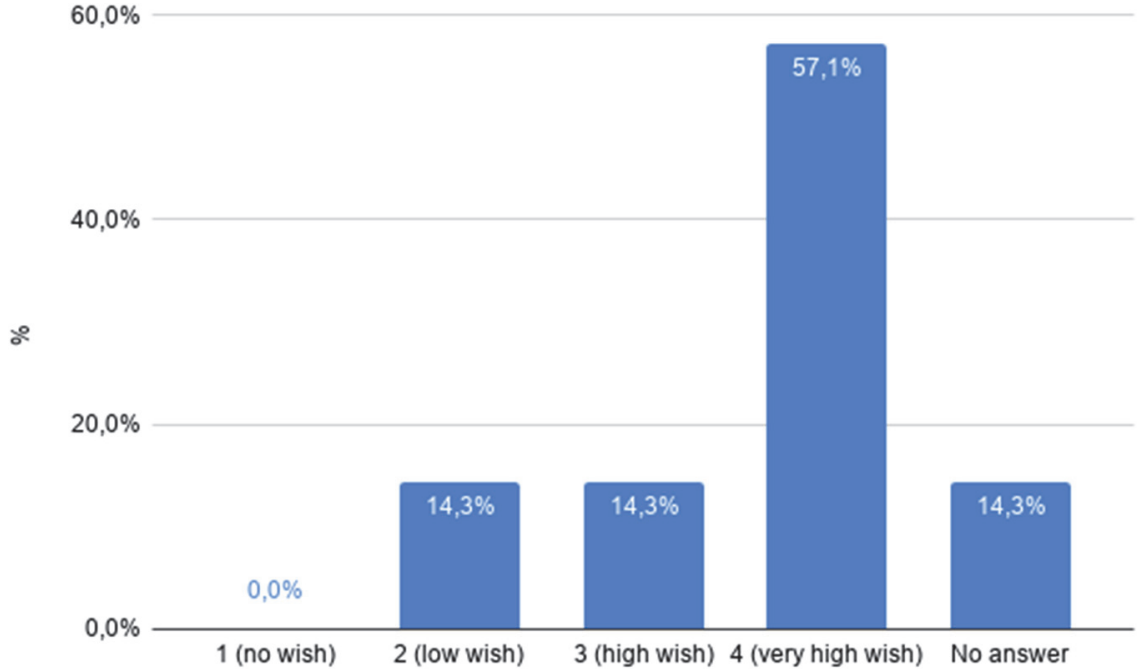

In a more problematised view, we can also highlight the acknowledgment made by someone that the residential conditions lived up to that moment were no longer appropriate or sustainable and, precisely, these reasons caused the reflection on one's current living conditions, lived and narrated in positive terms. The reason to leave the nuclear family context are multiple: the family may no longer exist; the structure of the relation with the nearest relatives may not be as deep and/or meaningful or, sometimes, if these relations exist, they are lived in a conflictual manner; because of age, the parent may not be any 
longer capable to offer support in line with the needs of the son or the daughter; the context of origin may cause impatience. In some cases, we can add to the above reasons a previous independent living experience that created unresolved problems, highlighting the inability to live in autonomy in terms of total selfmanagement and independence. In other cases, the declared well-being is based on the possibility of the project's response to the needs of safety (or containment of insecurity) of the person with disabilities, deemed - by him/her indispensable. Conversely, for example, in a situation where it was necessary to avoid the scission between the nuclear familial environment and a new living context, finding an apartment adjacent to the family's reference figure provided an alternative independent housing solution in line with the needs and wishes of the person and the family.

\section{Characteristics of the independent living projects}

The outcomes of the present survey on independent housing in Italy shed light on some characteristics of the housing projects currently active on the national territory, with a special focus on the ones developed in central and northern Italy. The housing services involved in this survey were firstly classified according to the duration of the hospitality, long or short-term, with a clear prevalence of the former $(78.3 \%)$, over the latter. It is an organizational model developed starting from the Seventies and well-rooted in the Italian social fabric; it arose in response to the previous forms of marginalization and separation to which people with disabilities were subjected (Cottini, 2009). On the other hand, short-term housing services host the people with disabilities for a short time, often for a weekend, a week or for some weeks during the year, for example, when one parent gets sick and the purpose is to give to the family the time to recover (a time of "relief", as defined one of the interviewed workers), or, when the person is gradually introduced to a wider experience of independence. In some cases, the apartment hosts different groups from week to week, accompanied by the presence of educators dedicated to developing specific domestic skills and competences.

The communities examined in the survey are small and composed of groups limited in the number of members, usually five or six persons, but sometimes even two or three, as well as experiences of independent living with larger groups, with cohabitation reaching ten persons. This orientation aimed at the development of small housing communities aims to improve the quality of life of the residents, offering the possibility for operators to ease interpersonal relationships and to enhance the paths of personal development, for the emergence of the needs and aspirations of each one and the structuring of the Life Project. 
The model for the housing services usually includes a high intensity of inhouse interventions, with educational (33.6\%), health $(29.1 \%)$ and voluntary personnel (18.2\%) present in the house at all times (41.3\%). More rarely, the services have a low intensity in their daily intervention $(15.2 \%$, from 1 to 5 hours), or medium-low (17.4\%, from 6 to 10) or medium-high (13.0\%, from 11 to 15 hours).

The limited presence of family members within these services (2.7\%) lends itself to different interpretations: it could be considered a priori an obstacle to the personal realisation of the family member or a destabilizing element with respect to the internal balances within the group of the inhabitants of the apartment, it is therefore discouraged; alternatively, it may derive from the option of the people with disabilities using these services not so much oriented to answer the needs of a progressive emancipation from the family context, as much as the demands of «those persons already unable to live in the family context for the fading away of the reference figures or their impossibility of taking care of the burden» (Cottini, 2009, p. 112), thus, ousting the family. In any case, this is a model of permanent residency to be overcome in order to structure extra-familiar living experiences "during us", rather than simply "after us" (Cottini, 2009). However, the wish to leave the nuclear family by the person with disabilities may provide a further reading of this analysis. $57.1 \%$ of the workers believe that the occupants of the services where they operate have "high" desire to leave the nuclear family to live independently. A meaningful variable begins thus to play a role, which - according to the perception of a subject- shows that the choice of the person to turn toward an independent housing perspective, demanded or accepted after a request, is perceived positively. This option may develop in terms of breaking away from the family context, as pointed out in some descriptions as this desire manifested itself, as well as in the process of maturation, emancipation and pursuit of the personal independence and together with the family. This maturation, built after experiencing family life, is enhanced when families are not isolated, but supported by appropriate educational and social support, favouring the entire family (Caldin and Cinotti, 2013).

\section{Pedagogical perspectives}

Residency models for people with disabilities no longer respond exclusively to health-related needs and to the possible need for assistance or support that the specific deficit requires, but are intentionally structured to offer ample spaces for relationships, opportunities, choice and personal fulfilment, acquisition of skills and competence for independence and emancipation.

When a person with disabilities grows up and becomes an adult, asks for 
his/her adulthood to be recognised in the family and in the society. The person's wish for emancipation towards new life experiences clearly emerges, starting from his/her home. Thus, living is presented as an element of social justice (the right to housing), as well as an element of maturation, personal fulfilment and social recognition of being an adult person.

In this scenario, it is essential to support the drafting and development of the Life Project together with the housing dimension, supporting the person with disabilities and the nuclear family in this planning process, through specific educational and social support.

\section{Bibliography}

Caldin R., Montuschi F., and Canevaro A. (2016). Il contributo culturale della Rivista "Studium Educationis" alla Pedagogia Speciale. Dimensioni concettuali e aree di ricerca. Studium Educationis, 2: 85-100.

Caldin R., and Cinotti A. (2013). Padri e figli/e disabili: vulnerabilità e resilienze. Studium Educationis, 3: 93-101.

Caldin R., and Friso V. (2012). Quale lavoro per le persone con disabilità, oggi, in Italia? Studium Educationis, 3: 37-57.

Cottini L. (2009). La famiglia e l'invecchiamento della persona con disabilità. In: Pavone M. (a cura di). Famiglia e progetto di vita. Crescere un figlio disabile dalla nascita alla vita adulta (pp. 103-123). Trento: Erickson.

D’Alonzo L. (2009). Il protagonismo del figlio disabile adulto: lavoro, università. In: Pavone M. (a cura di), Famiglia e progetto di vita. Crescere un figlio disabile dalla nascita alla vita adulta (pp. 79-97). Trento: Erickson.

Edgar W., and Meert H. (2005). Fourth Review of Statistics on Homelessness in Europe. The ETHOS Definition of Homelessness. Brussels: FEANTSA.

Edgar W., Meert H., and Doherty J. (2004). Third Review of Statistics on Homelessness in Europe. Developing an Operational Definition of Homelessness. Brussels: FEANTSA.

FEANTSA, Abbé Pierre Foundation (2019). Fourth Overview of Housing Exclusion in Europe 2019.

Friso V. (2017). Disabilità, Rappresentazioni sociali e Inserimento Lavorativo. Percorsi identitari, nuove progettualità. Milano: Guerini.

Goussot A. (a cura di) (2009). Il disabile adulto. Anche i disabili diventano adulti e invecchiano. Rimini: Maggioli editore.

Lepri C. (2011). Viaggiatori inattesi. Appunti sull'integrazione sociale delle persone disabili. Milano: FrancoAngeli.

The United Nations [UN] (2006). Convention on the Rights of Persons with Disabilities. The United Nations.

World Health Organization [WHO] (2001). International classification of functioning, disability and health: ICF. World Health Organization. 